\title{
Yeast Exoglycoproteins Produced Under NaCl-Stress Conditions as Efficient Cryoprotective Agents
}

\section{E. Breierova}

Culture Collection of Yeasts, Institute of Chemistry, Slovak Academy of Sciences, Dubravska cesta 9, 84238 Bratislava, Slovakia. E-mail: chememi@savba.sk.

\begin{abstract}
Six extracellular yeast glycoproteins were prepared from three yeast species in osmotic equilibrium and unequilibrium environments and used as non-penetrating cryoadditives. Glycoproteins secreted by the strain Dipodascus australiensis into growth medium containing $\mathrm{NaCl}(8 \% \mathrm{w} / \mathrm{v})$ were found to be the most effective cryoadditives. It was possible to use these glycoproteins alone (without DMSO as penetrating agent) for the cryoprotection of the studied yeasts.
\end{abstract}

\section{INTRODUCTION}

Many factors that affect the successful freeze preservation of yeast cells have been documented (1). The crucial problem during preservation is to ensure genetic stability. The viability of the cells after preservation may correlate with the extent of protection against dehydration stress and with the transition of water to ice crystals $(2,3)$. Survival and stability of all strains preserved in liquid nitrogen ($196^{\circ} \mathrm{C}$ ) is always higher in the presence of a mixture of penetrating and non-penetrating cryoprotective agents (4) than in the presence of non-penetrating cryoagents only (5). The basic feature of nonpenetrating cryoprotective agents is the ability of their hydrophilic groups to bind water, giving rise to a phenomenon often described as "bound water" (6). In higher cells, glycoproteins have various functions such as surface receptors, cell-cell mediators, components of the extracellular matrix, cryoprotectors, etc. The protein moiety of most glycoproteins is the functional part, while the carbohydrate moiety contributes to the attainment of an adequate tertiary structure and modifies the glycoprotein molecule making it more resistant to degradation, and facilitates its secretion (7).

This paper describes the cryoprotective activity of six extracellular yeast glycoproteins prepared from three yeast species cultivated under two different conditions.

\section{MATERIALS AND METHODS}

\section{Yeast strains}

The following psychrophilic species were used: Candida graminis CCY 29-133-1, Candida capsuligena

(C)1998 Biological Procedures Online. All rights reserved. Paper-based copying permitted for internal use for educational or non-profit purposes only. Otherwise, this article may be copied to paper provided that \$US15 per copy is paid directly to Biological Procedures Online, GMO 106 Box 44, Waterloo ON, Canada N2L 3G1. Electronic copying, storage or redistribution prohibited. ISSN: 1480-9222

Biological Procedures Online • Vol. 1 No. $1 \bullet$ May 14, $1998 \bullet$ www.biologicalprocedures.com 
CCY 29-143-1, Leucosporidium scottii CCY 64-1-1, Phaffia rhodozyma CCY 77-1-1, Leucosporidium antarcticum CCY 64-3-1, Candida diffluens CCY 29-132-1, Sympodiomyces parvus CCY 73-1-1, Dioszegia hungarica CCY 18-1-1, Candida frigida CCY 29-144-1, Candida gelida CCY 29-121-1. All yeast strains were obtained from the Culture Collection of Yeasts (Institute of Chemistry, SAS, Bratislava, Slovakia).

\section{Basal cryoprotective medium}

Basal cryomedium consisted of $7 \mathrm{~g}$ wort extract, $0.3 \mathrm{~g}$ yeast extract (Difco), $0.5 \mathrm{~g}$ peptone (Difco) per $100 \mathrm{ml}$ of tap water. The medium was three times sterilized by heating at $100^{\circ} \mathrm{C}$ for $1 \mathrm{~h}$ on three separate days.

\section{Cryoadditives}

After sterilization of the basal cryomedium, DMSO (Fluka, Switzerland) was added as a penetrating cryoagent to give a final concentration of $10 \%(\mathrm{v} / \mathrm{v})$ in the first series of cryomedia. The second series was prepared without DMSO. All cryomedia contained the crude ethanol precipitates of the different extracellular yeast glycoproteins of three yeast species. These non-penetrating cryoagents were added before sterilization into cryomedia to give a final concentration of $0.27 \%$.

\section{Cultivation conditions for preparation of the extracellular yeast glycoproteins}

Control condition optimal mineral cultivation medium with $2 \%$ glucose contained per $1: 4 \mathrm{~g}$ yeast extract, $10 \mathrm{~g}\left(\mathrm{NH}_{4}\right)_{2} \mathrm{SO}_{4}, \quad 1 \mathrm{~g} \quad \mathrm{KH}_{2} \mathrm{PO}_{4}, \quad 0.2 \mathrm{~g} \quad \mathrm{~K}_{2} \mathrm{HPO}_{4}, \quad 0.1 \mathrm{~g} \quad \mathrm{NaCl}, 0.1 \mathrm{~g} \mathrm{CaCl}_{2}, 0.5 \mathrm{~g} \quad \mathrm{MgSO}_{4}$, and $1 \mathrm{ml}$ microelements solution $\left(1.25 \mathrm{~g} \mathrm{H}_{3} \mathrm{BO}_{3}, 0.1 \mathrm{~g} \quad \mathrm{CuSO}_{4}, 0.25 \mathrm{~g} \mathrm{KI}, 1 \mathrm{~g} \mathrm{MnSO}_{4}, 0.5 \mathrm{~g} \quad \mathrm{FeCl}_{3}, 0.5 \mathrm{~g}\right.$ $\left(\mathrm{NH}_{4}\right)_{6} \mathrm{Mo}_{7} \mathrm{O}_{24}, 1 \mathrm{~g} \mathrm{ZnSO}$ contained per 1). The optimal cultivation temperatures used were $28^{\circ} \mathrm{C}$ for Dipodascus australiensis and $17^{\circ} \mathrm{C}$ for Waltomyces kononenkoe and $W$. lipofer (Table 1). The cells were precultured aerobically in the test tubes to the stationary phase of growth and then cultivated on a reciprocal shaker to the stationary phase in 11 flasks with $500 \mathrm{ml}$ of optimal mineral medium. After centrifugation crude extracellular glycoproteins were isolated by precipitation from the supernatant by adding two volumes of $96 \%(\mathrm{v} / \mathrm{v})$ ethanol and subsequent centrifugation. The ethanol precipitate was dissolved in distilled water, dialyzed against distilled water and freeze-dried.

To produce the stress conditions, 4\% (W. kononenkoe, W. lipofer) and 8\% $\mathrm{NaCl}$ (D. australiensis), was added to the control medium.

\section{Preservation in liquid nitrogen}

The cell suspension $\left(0.1 \mathrm{ml}, 10^{7}-10^{8}\right.$ cells in $\left.1 \mathrm{ml}\right)$ and cryoprotective medium $(0.5 \mathrm{ml})$ were pipetted into sterile 2-ml polyethylene ampoules (Koh-i-noor, Dalecin), and rapidly frozen in liquid nitrogen (no later than $1 \mathrm{~h}$ after pipetting). Three ampoules were used for each strain as well as cryomedium. Cultures had been stored for one year. After 1 year of storage in liquid nitrogen, the cultures were thawed in a $37^{\circ} \mathrm{C}$ water bath for $30 \mathrm{~min}$. opened, and recultivated. 


\section{Survival of the storage culture}

Qualitative evaluation was performed by visual screening of growth on wort agar after spreading. Growth was evaluated by 1-3 crosses for the culture grown taking into account optimal time of growth ( 1 cross $=$ weak growth, 2 crosses $=$ medium growth, 3 crosses $=$ good growth $)$; the cultures could maximally reach 3 crosses $=100 \%$.

Table 1. Designation of the glycoproteins described in the paper

\begin{tabular}{|c|c|c|c|c|}
\hline \multirow{3}{*}{$\begin{array}{l}\text { Strains } \\
\text { Name }\end{array}$} & & \multicolumn{3}{|c|}{ Glycoprotein designation } \\
\hline & \multirow{2}{*}{$\begin{array}{l}\text { No. } \\
\text { CCY }\end{array}$} & \multicolumn{3}{|c|}{$\% \mathrm{NaCl}$ in cultivation medium } \\
\hline & & 0 & 4 & 8 \\
\hline Dipodascus australiensis & $52-6-1$ & $\mathrm{G}_{1}$ & NG & $\mathrm{G}_{2}$ \\
\hline Waltomyces kononenkoe & $33-4-2$ & $\mathrm{G}_{3}$ & $\mathrm{G}_{4}$ & NG \\
\hline Waltomyces lipofer & $33-2-4$ & $\mathrm{G}_{5}$ & $\mathrm{G}_{6}$ & NG \\
\hline
\end{tabular}

NG, not grown.

\section{RESULTS AND DISCUSSION}

The six crude glycoproteins were tested to determine their effect on the survival of the 10 psychrophilic yeast strains stored in liquid nitrogen.

The strains could be divided into three groups on the basis of their survival rates in the basal medium containing DMSO (8) (Figs. 1, 2). The strains survived only to a limited extent (to 4\%) or did not survive in the basal medium without any cryoadditive.

Group I comprised strains with the highest survival rates $(20 \pm 6 \%)$. These strains were typical psychrophilic species. Each of them produced some protective agent such as carotenoid pigment or slime. The most suitable glycoprotein cryoprotectants were $\mathrm{G}_{2}, \mathrm{G}_{4}$ and $\mathrm{G}_{6}$ produced under $\mathrm{NaCl}-$ induced stress conditions. Without DMSO (Figs. 1, 2) the most effective cryomedium was the one containing The average survival rate was about $75 \%$.

Group II comprised four strains with lower survival rates (about $15 \pm 5 \%$ ). The strains of $D$. hungarica and $C$. gelida survived somewhat, while $C$. graminis and $L$. scottii survived to the lesser extent.

Group III comprised the most cryosensitive strains (survival rates 0 - 5\%).

In the DMSO-free cryomedium only small differences in the percentage of survival when using various glycoproteins were observed. The glycoproteins produced by the strains of $D$. australiensis under stress conditions ( $8 \% \mathrm{NaCl}$ in the cultivation medium) had the highest influence on the cryosensitive psychrophilic yeast species (Figs. 1, 2). The glycoprotein G2 was the most effective for all strains. The order of the effectivity of glycoproteins wass $>\mathrm{G}_{1}>\mathrm{G}_{6}>\mathrm{G}_{5}>\mathrm{G}_{3}>\mathrm{G}_{4}$.

The extracellular yeast glycoproteins produced by the genus Waltomyces $\left(\mathrm{G}_{3}, \mathrm{G}_{4}, \mathrm{G}_{5}\right.$ and $\left.\mathrm{G}_{6}\right)$ were not very effective as cryoprotective agents. The ability of these cultures to grow at the higher concentration of $\mathrm{NaCl}$ is probably dependent on the structure of the glycoproteins produced. During freezing, the 
residual solution becomes hypertonic with regard to the intracellular compartment, and thus the cells are subjected to a fast decrease of osmotic pressure. The glycoproteins isolated from the $8 \% \mathrm{NaCl}$ medium were efficient as cryoprotective agents.

The results of the study of the survival rates will be used to develop experimental protocols that minimize osmotic stress without the penetrating cryoprotectant DMSO. The application of the above results to another type of eucaryotic cells has also been described (9). 
Group I (with DMSO):

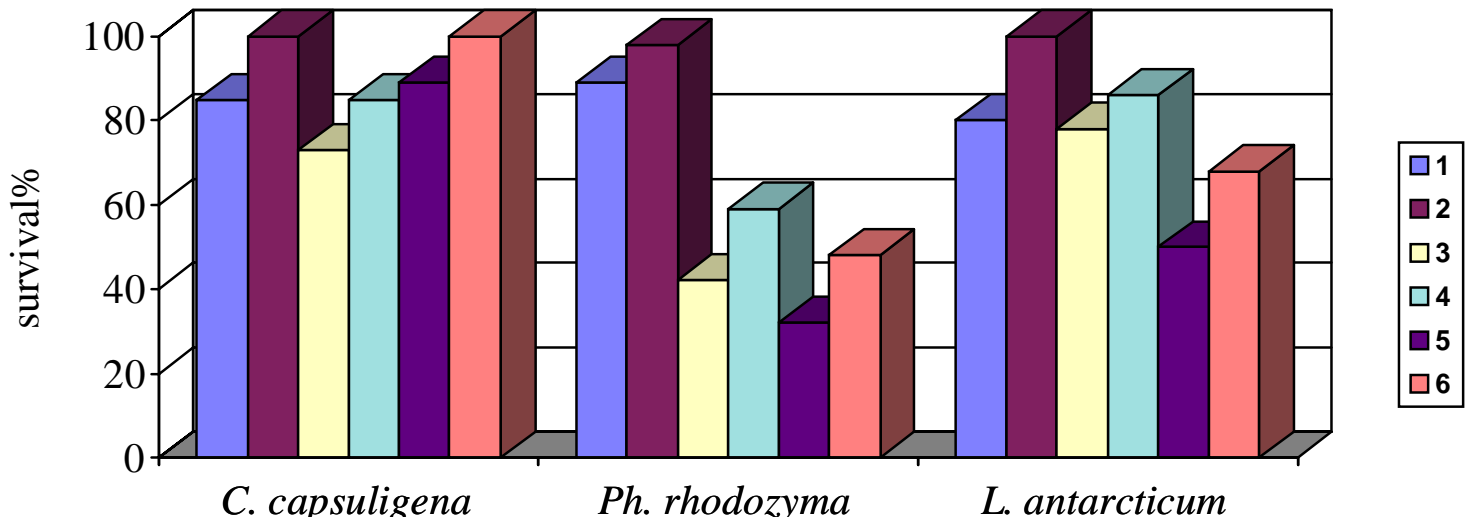

Group I (without DMSO):

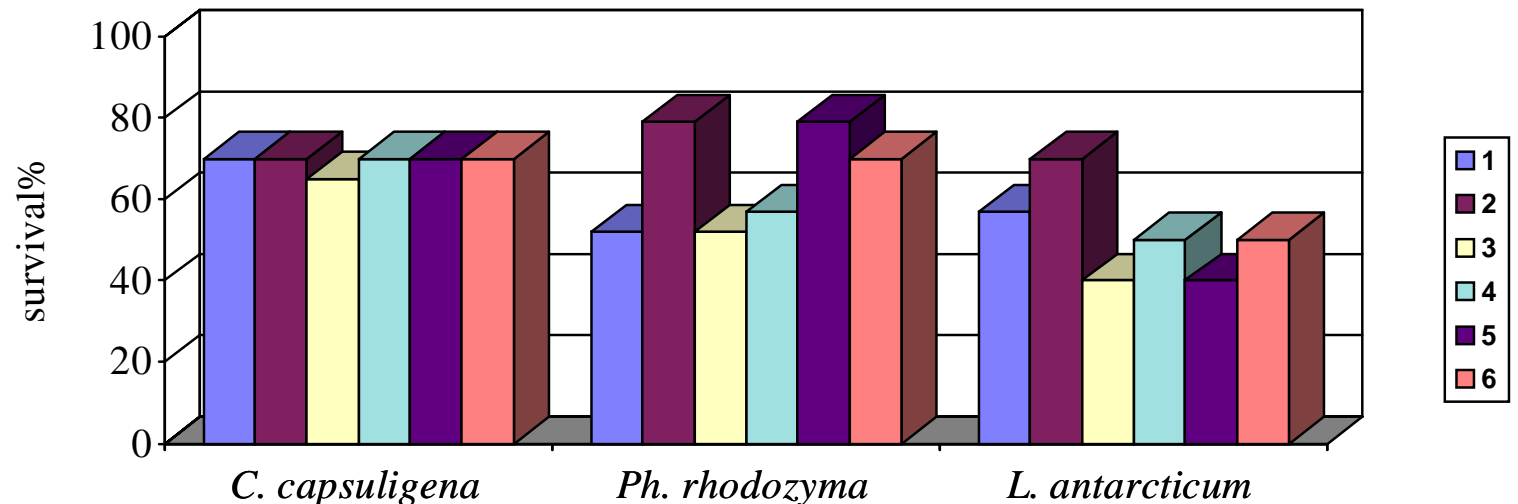

Group II (with DMSO):

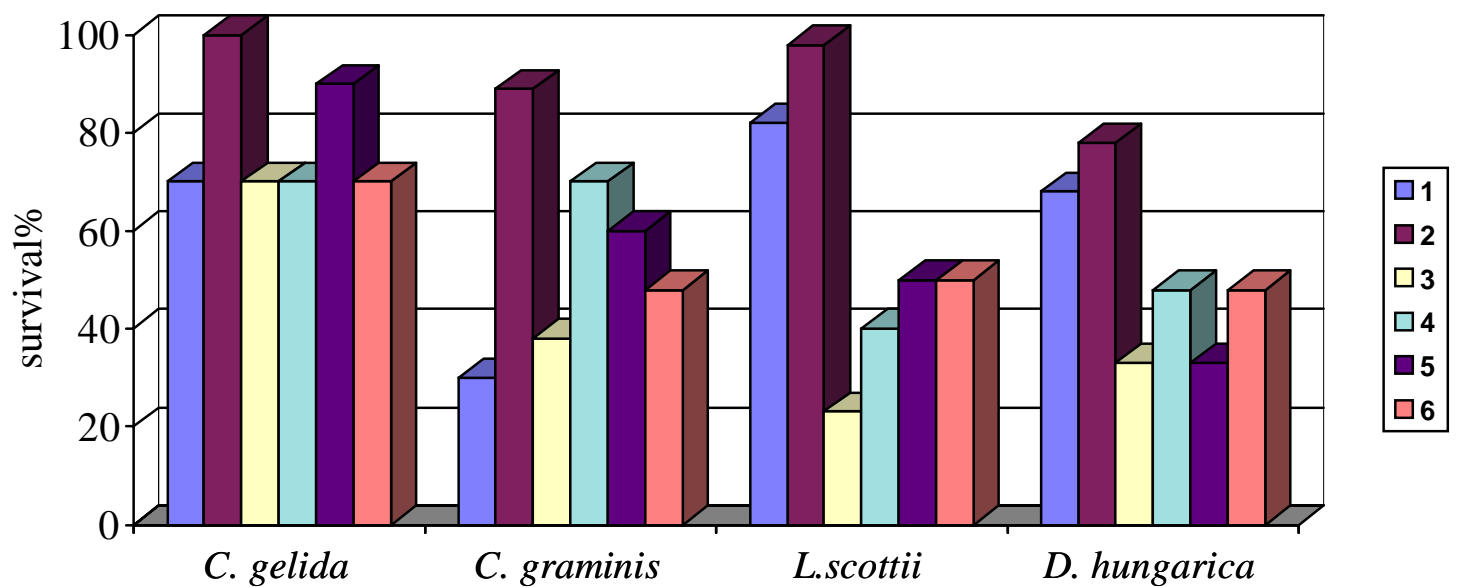

Fig. 1. Survival of the strains tested depending on the combination of basal medium (BM) and nonpenetrating cryoagents with and without DMSO. $1, \mathrm{BM}+\mathrm{G}_{1} ; 2, \mathrm{BM}+\mathrm{G}_{2} ; 3, \mathrm{BM}+\mathrm{G}_{3} ; 4, \mathrm{BM}+\mathrm{G}_{4} ; 5$, $\mathrm{BM}+\mathrm{G}_{5} ; 6, \mathrm{BM}+\mathrm{G}_{6}$. Data represent the mean percentage from three independent experiments 
Group II (without DMSO):

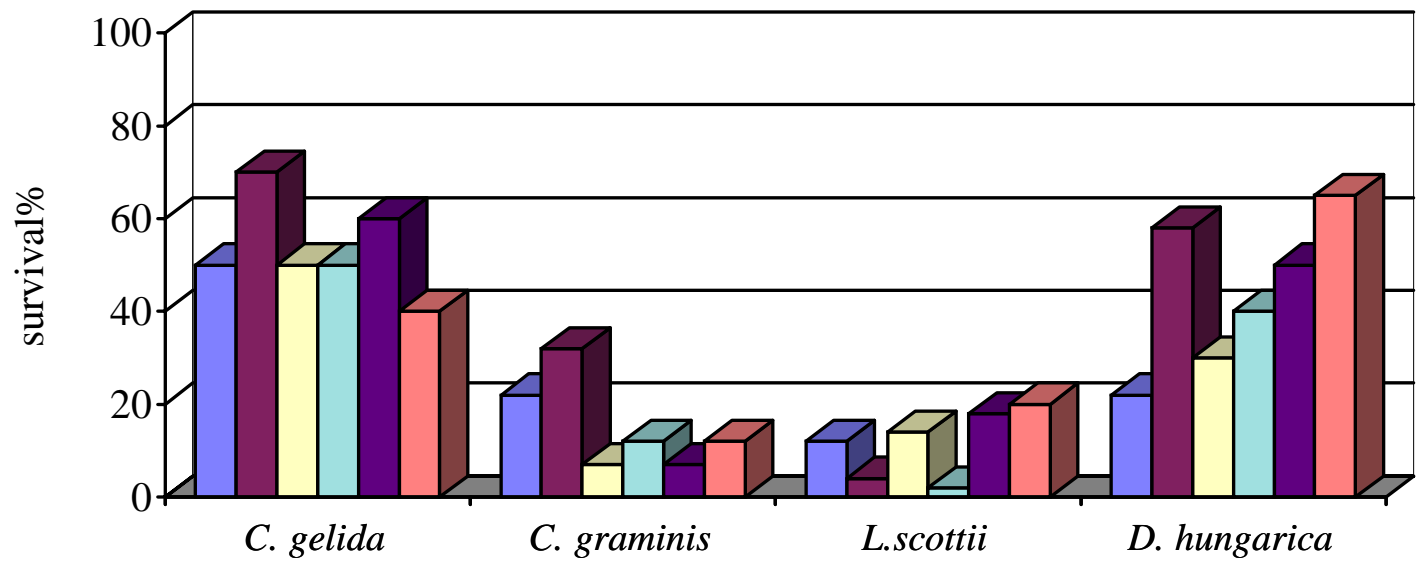

Group III (with DMSO):

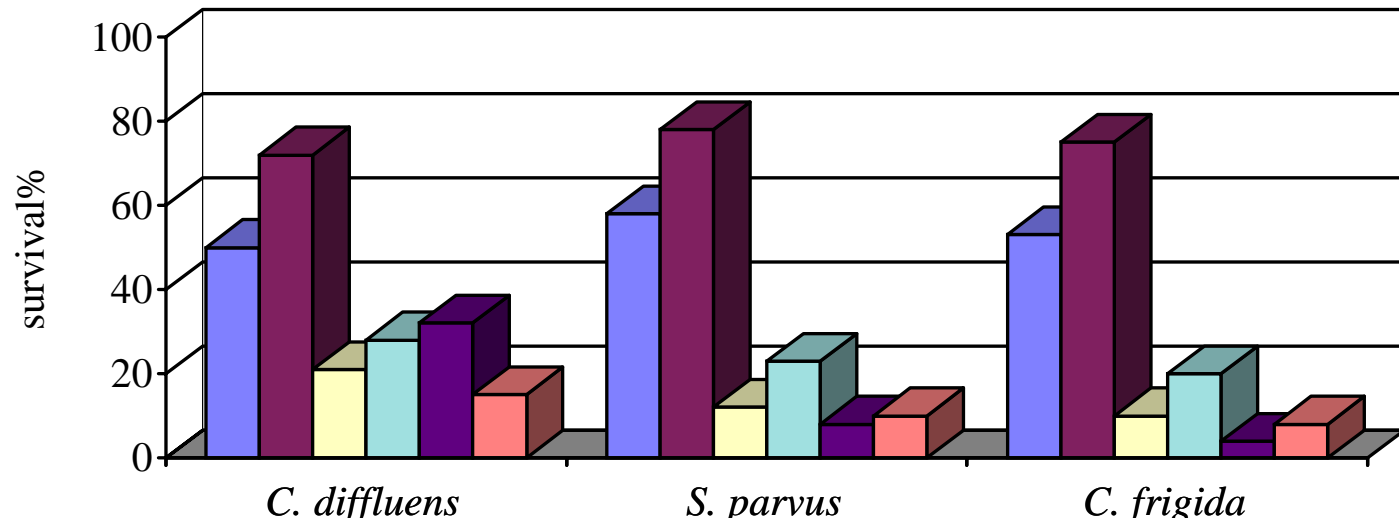

Group III (without DMSO):

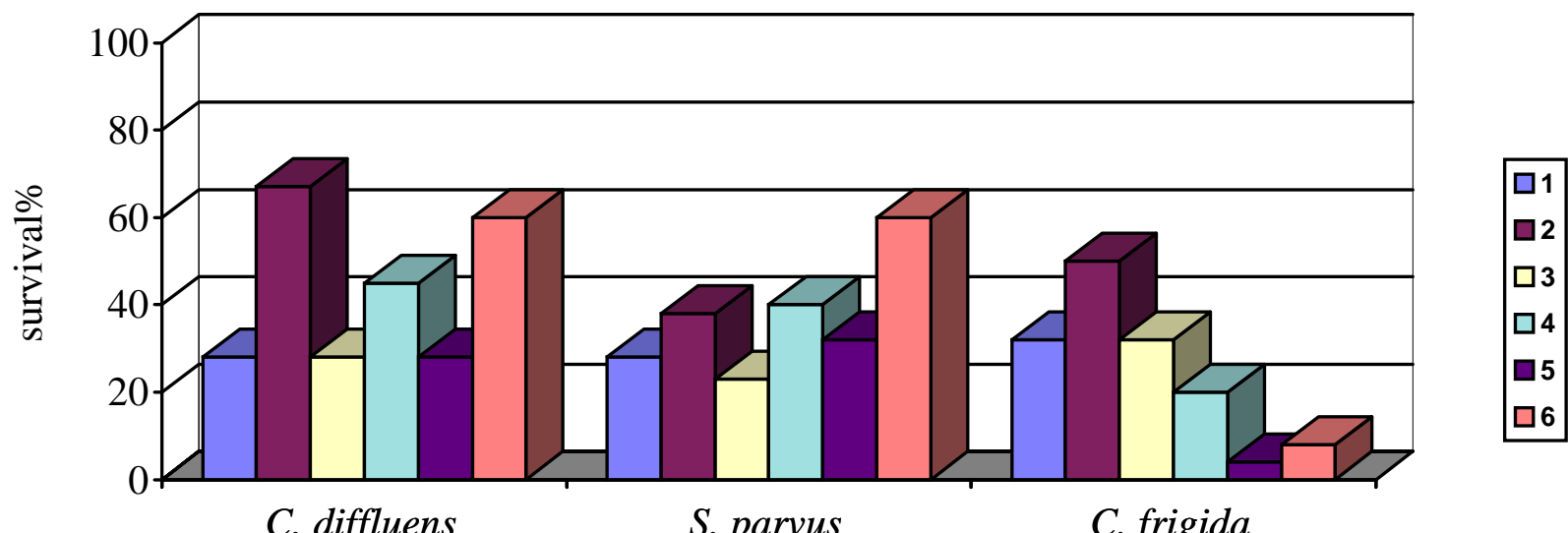

Fig. 2. Fig. 1 cont... Survival of the strains tested depending on the combination of basal medium (BM) and nonpenetrating cryoagents with and without DMSO. $1, \mathrm{BM}+\mathrm{G}_{1} ; 2, \mathrm{BM}+\mathrm{G}_{2} ; 3, \mathrm{BM}+\mathrm{G}_{3} ; 4, \mathrm{BM}+\mathrm{G}_{4} ; 5$, $\mathrm{BM}+\mathrm{G}_{5} ; 6, \mathrm{BM}+\mathrm{G}_{6}$. Data represent the mean percentage from three independent experiments 


\section{ACKNOWLEDGEMENT}

The author gratefully acknowledges the support from VEGA grant number 2/4145.

\section{REFERENCES}

1. Nakagawa S., Ouchi K. 1994. Improvement of freeze tolerance of commercial baker's yeasts in dough by heat treatment before freezing. Bioscience, Biotechnology and Biochemistry 11, 2077 2079.

2. Berendsen H. J. C. 1975. Water a comprehensive treatise. F. Franks, New York: Plenum, 293-330, 5

3. Tan C. S., Van Ingen C.S., Talsma M. et al. 1995. Freeze-drying of fungi: influence of composition and glass transition temperature of the protectant. Cryobiology 32, 60-67.

4. Breierova E., Kockova-Kratochvilova A. 1992. Cryoprotective effects of yeast extracellular polysaccharides and glycoproteins. Cryobiology 29, 385-390.

5. Korber Ch., Scheiwe M. W., Boutron P. 1982 Can the stability of the completely amporphous state explain the cryoprotection of hydroxyethyl starch? Cryo-Letters 3, 83-90.

6. Franks F. 1982. Apparent osmotic activities of water soluble polymers used a cryoprotectant. CryoLetters 3, 115-120.

7. Tanner W. Lehle L. 1987. Protein glycosylation in yeast. Biochimica et Biophysica Acta 906, 81-89.

8. Breierova E. 1994. Cryoprotection of psychrophylic yeast species by the use of additives with cryoprotective media. Cryo-Letters 15, 191-197.

9. Tilser J., Breierova E., Tichy M., et al. 1996. Effect of supplementation of University of Wisconsin solution with glycoproteins from psychrophilic strains of yeast on hypothermic liver storage of rats. Cryobiology 33, 347-353. 